menon of the cool flame, sensitization and inhibition of ignition and pyrolysis of paraffins.

This book can certainly be recommended to all chemists; it provides an easily read account of so many important fields of study. It is well provided with references and illustrated by clear diagrams. The editors and authors have provided their former professor with a tribute which, I am sure, must give him great pleasure and which justly matches his own reputation.

JAMES C. ROBB

\section{CHEMISTRY OF COAGULATION}

\section{Blood Clotting Enzymology}

Edited by Walter H. Seegers. Pp. xii +628 . (New York: Academic Press, Inc.; London: Academic Press, Inc. (London), Ltd., 1967.) 2208.

Is a sense this book is disappointing in that it does not, as its title suggests, devote much space to the enzymology of blood coagulation; instead this is a comprehensive review of the mechanism of blood coagulation from the particular point of view associated with Dr Seegers. It is unfortunate that the author has not given more space to comparing and contrasting his own view with that pro. posed by other workers, which he dismisses with the phrase ". . . it is speculative in nature and proposes a sequence of events that is not consistent with the known facts of prothrombin chemistry". It is, in fact, the chemistry of prothrombin which is at the very centre of the discord between Seegers and those other "speculators". He believes that prothrombin isolated by procedures laid down by him is a multifunctional complex which is dissociated into separate activities by thrombin. Other authors believe that his prothrombin preparations are a mixture of proteins, and have supported this belief by resolving the complex, by various procedures, into different components of which the precursor of thrombin is only one. Seegers claims that in these experiments the prothrombin has been modified and no longer represents the native substance.

The individual view of Seegers and his associates is apparent in the chapters devoted to the clinical aspects of coagulation and to immunochemistry, but the book also includes contributions from other authors on topics about which Seegers holds no particular view. There is an excellent review on the fibrinogen fibrin transformation incorporating the latest ideas on the mechanism of fibrin polymerization, and an interesting chapter on the ultrastructure of the fibrin clot. There is also a stimulating discussion on the effect of different surfaces on the proteins involved in blood coagulation.

Other aspects of coagulation which are included are the various inhibitors of blood coagulation, the role of platelets in haemostasis and, finally, a short account of the chemistry and function of vitamin $K$.

In spite of these reservations, the student of blood coagulation should find this book useful because it presents an alternative interpretation of the phenomena of clotting, the merits of which can be decided by the reader.

M. P. Esnour

\section{ANTIBODIES FOR CLOTTING}

The Use of Antibodies in the Study of Blood Coagulation By K. W. E. Denson. Pp. ix + 244. (Oxford and Edinburgh: Blackwell Scientific Publications, 1967.) 52s.6 6 . net.

THE mysteries which surround blood coagulation are such as to make the development of a new method for its study an important event. In recent years the addition of techniques of protein chemistry, particularly electrophoresis and ultracentrifugation, to the long-established coagulation methods has provided new information about the physical characteristics of certain clotting factors. The present volume describes a new approach to the study of the coagulation mechanism, through the provision and elaboration of a technique, which may prove a useful addition to those already mentioned.

The work described in this book was originally submitted as a thesis for a DPhil degree in the University of Oxford and is essentially the application of immunological methods, in the form of the use of antisera to clotting factors, to the study of blood coagulation.

The book is in two parts. The first part begins with a description of the two modern concepts of blood coagulation, the "cascade" hypothesis and that which envisages prothrombin as the basic factor, and continues with a detailed description of methods for preparing purified coagulation factors and thus their antisera. There follow two chapters on the characterization of the functional activity of the antisera of six clotting factors and one chapter dealing with the immunochemistry of clotting factors and their antisera.

The second part of the book describes, in great detail, the application of the specific antisera to clotting factors to various aspects of the coagulation mechanism and ends with a general discussion, including the author's conclusions and suggestions for further studies.

The detail in which the methods and experiments are described and the results presented is impressive and the treatise is obviously the result of a vast amount of detailed and painstaking labour, on which the author is to be congratulated.

This volume will be of greater value to those wishing to apply these techniques and procedures to their own investigations than to those with a more general interest in coagulation, although the summaries and conclusions at the end of most chapters will assist those not immedi. ately concerned with the full experimental details, while the review of the literature is adequate for the needs of the treatise.

The approach described in this treatise has undoubtedly added, and will continue to add, to our knowledge of the coagulation mechanism. While it may help to resolve some of the differences between the "cascade" hypothesis, founded as it is on investigation of defects in the haemostatic mechanism, and that based on prothrombin, it remains to be seen whether its application will enable new. light to be thrown on the all-important and pressing problem of hypercoagulability and thromboembolic disease.

G. B. D. ScotT

\section{IMMUNOLOGICAL EXPLOSION}

\section{Advances in Immunology}

Edited by F. J. Dixon, jun., and J. H. Humphrey. Vol. 6. Pp. xvii +571. (New York: Academic Press, Inc.; London: Academic Press, Inc. (London), Ltd., 1967 ) $148 s$. THIs volume contains a varied selection of topics. Many of the articles, however, have the common feature that they report results which appear to be just opening the way to the most productive phases of study. For example. P. G. H. Gelland A. Kelus in their article on anti-antibodies devote two pages to idiotypic antibodies. This is a most remarkable phenomenon first observed independently several years ago by Oudin and by the authors, and the far-reaching theoretical implications have aroused such interest that a fuller discussion will be needed very soon. Again the in vitro stimulation of a primary response has been sought for many years and much of Dutton's review is taken up with discussion of unsuccessful or ancillary studies related to this basic problem. The most convincing demonstration that a primary stimulation in vitro was possible, by Mishell and Dutton, must have been made: while this review was in preparation and of necessity gets 